\begin{tabular}{|l|l|l||}
\hline \multicolumn{2}{|c|}{ PublisherInfo } \\
\hline \hline PublisherName & $:$ & BioMed Central \\
\hline \hline PublisherLocation & $:$ & London \\
\hline \hline PublisherImprintName & $:$ & BioMed Central \\
\hline \hline
\end{tabular}

\title{
Isoflurane - induced myocardial preconditioning
}

\begin{tabular}{|l|l|l||}
\hline \multicolumn{2}{|c||}{ ArticleInfo } \\
\hline \hline ArticleID & $:$ & 4114 \\
\hline \hline ArticleDOI & $:$ & $10.1186 /$ ccf-1999-341 \\
\hline \hline ArticleCitationID & $:$ & 341 \\
\hline \hline ArticleSequenceNumber & $:$ & 51 \\
\hline \hline ArticleCategory & $:$ & Paper Report \\
\hline \hline ArticleFirstPage & $:$ & 1 \\
\hline \hline ArticleLastPage & $:$ & 4 \\
\hline \hline & & RegistrationDate : 1999-5-24 \\
\hline ArticleHistory & $:$ & OnlineDate \\
\hline \hline ArticleCopyright & $:$ & Current Science Ltd1999-5-24 \\
\hline \hline ArticleGrants & $:$ & \\
\hline \hline ArticleContext & $:$ & 130541111 \\
\hline \hline
\end{tabular}




\section{Keywords}

Ischemia, isoflurane, KATP channel, myocardial preconditioning, protection

\section{Comments}

This paper presents powerful evidence that isoflurane provides myocardial protection in a similar fashion to ischemic preconditioning via actions on adenosine and KATP channels. This effect may be different in rabbits compared to humans due to difference in blood pressures, heart rates and myocardial mass, however, the model provides evidence of reduction of infarct size with ischemic preconditioning which has been seen in human preparations. Most importantly this study used clinically relevant doses of isoflurane (0.6 MAC) for a duration of $15 \mathrm{~min}$. This evidence suggests that isoflurane or other halogenated ethers are the anesthetic agents of choice for patients at risk of myocardial ischemia and may also be advantageous for sedating such patients on intensive care units.

\section{Introduction}

Myocardial preconditioning refers to the ability of short periods of myocardial ischemia to provide subsequent myocardial protection against prolonged ischemia. This finding has potential for improving strategies for myocardial protection in patients with ischemic heart disease undergoing both cardiac and non-cardiac surgery. Pharmacological methods of providing myocardial preconditioning are currently unsatisfactory. Available agents known to induce myocardial preconditioning (adenosine, cromokalin, and acetylcholine) must be adminstered into the coronary sinus, and they cause unacceptable cardiovascular side effects. Halogenated anaesthetic agents have been shown to mimic the myocardial preconditioning effect and reduce infarct size. The receptor mechanism for this effect is not characterised in full, but adenosine receptors and potassium channels have been implicated.

\section{Aims}

The aim of this study was to test the hypothesis that the myocardial preconditioning like effect of isoflurane is mediated by KATP channels. 


\section{Methods}

New Zealand white rabbits were anesthetised, ventilated to normocapnia and had their carotid arteries cannulated to allow arterial blood gas analysis. Electrocardiogram (ECG) and end tidal carbon dioxide tensions were measured. Median sternotomy was performed and a snare placed around the animals anterolateral coronary artery. Occlusion of the artery was confirmed with ECG changes and regional cyanosis. After placing the snare the rabbits were randomised into one of seven groups . All animals underwent $30 \mathrm{~min}$ of coronary artery occlusion followed by $3 \mathrm{~h}$ of reperfusion.

The groups were as follows:(1) No pretreatment (controls); (2) Ischemia preconditioning, 5 min of occlusion, $15 \mathrm{~min}$ of reperfusion then 30 min occlusion; (3) Isoflurane preconditioning, $15 \mathrm{~min}$ of 0.6 MAC isoflurane followed by $15 \mathrm{~min}$ washout, then $30 \mathrm{~min}$ occlusion; (4) IV glyburide $0.33 \mathrm{mg} / \mathrm{kg}$ given $25 \mathrm{~min}$ before isoflurane; (5) IV 8-(p-sulfophenyl)-theophylline (SPT) $7.5 \mathrm{mg} / \mathrm{kg}$ given $25 \mathrm{~min}$ before isoflurane; (6) IV glyburide $0.33 \mathrm{mg} / \mathrm{kg}$ but no isoflurane; (7) IV SPT $7.5 \mathrm{mg} / \mathrm{kg}$ but no isoflurane.

Experimental protocols were performed after a period of 20-25 min stability post surgical incision. The heart was stopped with an anesthetic overdose and the heart excised to identify the area at risk and infarct size. Area at risk (AR) was determined by occluding the coronary artery of the excised heart (as per the experimental protocol) and perfusing the heart with flourescent microspheres. The AR was then the portion of the heart not illuminated with ultraviolet (UV) light. The infarcted area was stained using the vital stain triphenyltetrazolium chloride and the area traced out. AR and infarct size were calculated in grammes from slice area and mass.

\section{Results}

Group sizes were as follows: controls 13; ischemia preconditioned 10; isoflurane pretreatment 9; isoflurane and glyburide 10; isoflurane and SPT, 8 and 7 each in the glyburide and SPT groups. Five rabbits died of intractable ventricular fibrillation after coronary occlusion (three controls, one ischemic preconditioning, one isoflurane) and two developed intractable hypotension (both in the isoflurane and glyburide group). Ratio of infarct size to area at risk (IS:AR) were comparable in all seven groups. IS:AR was $30.2 ? 11 \%$ in the control group and significantly reduced with ischemic preconditioning $(8.3$ ? 5\%) and isoflurane pretreatment (13 ? 8.2\%). The infarct size in the other groups did not differ significantly from the controls. There were no differences in hemodynamic variables between the groups.

\section{Discussion}

The results of this study suggest that pretreatment with a clinically relevant dose of isoflurane can reduce infarct size. This isoflurane mediated protection is reduced by the concomitant administration of 
the KATP channel inhibitor (glyburide) or adenosine receptor anatgonists (SPT). Similar myocardial protection has previously been demonstrated with all the other halogenated vapours in current useage. The mechanism by which isoflurane interacts with the KATP channel is not clear. The only evidence of direct action on the channel is to induce closure; however, in vivo isoflurane seems to act to open the channel. It may be that isoflurane acts to desensitise the KATP channel to ATP thereby allowing channel opening at higher ATP levels. This change in channel sensitivity may be caused directly or indirectly by intracellular mechanisms. The other mechanism may be via $\mathrm{G}$ protein or proteinkinase $\mathrm{C}$. Potential flaws of the study discussed include the fact that propofol infusion rates were reduced in the isoflurane group but this should not have led to improved myocardial protection as propofol itself has myocardial protective properties.

\section{References}

1. Ismaeil MS, Tkachenko I, Gamperi AK, Hickey R, Cason B: Mechanisms of Isoflurane-induced myocardial preconditioning in rabbits. Anesthesiology. 1999, 90: 1812-1821. 